\title{
Correspondence between formulations of Avrami and Gompertz equations for untreated tumor growth kinetics
}

\author{
N. A. Villar Goris \\ Universidad Autónoma de Santo Domingo, Santo Domingo, Dominican Republic. \\ Universidad Católica del Cibao, La Vega, Dominican Republic. \\ Centro Nacional de Electromagnetismo Aplicado, Universidad de Oriente, Santiago de Cuba, Cuba.
}

\author{
A. R. Selva Castañeda \\ Dependencia y dirección Departamento de Telecomunicaciones, Facultad de Ingeniería en Telecomunicaciones, \\ Informática y Biomédica, Universidad de Oriente, Santiago de Cuba, Cuba. \\ E. E. Ramirez-Torres \\ Departamento de Biomédica, Facultad de Ingeniería en Telecomunicaciones, \\ Informática y Biomédica, Universidad de Oriente, Santiago de Cuba, Cuba.
}

\author{
J. Bory Reyes \\ ESIME-Zacatenco. Instituto Politécnico Nacional, México.
}

\author{
L. Randez \\ Instituto Universitario de Investigación de Matemáticas y Aplicaciones, Universidad de Zaragoza, Zaragoza, España. \\ L. E. Bergues Cabrales \\ Centro Nacional de Electromagnetismo Aplicado, Universidad de Oriente, Santiago de Cuba, Cuba. \\ Instituto Universitario de Investigación de Matemáticas y Aplicaciones, Universidad de Zaragoza, Zaragoza, España. \\ e-mail: berguesc@yahoo.com, \\ J. I. Montijano \\ Instituto Universitario de Investigación de Matemáticas y Aplicaciones, Universidad de Zaragoza, Zaragoza, Spain \\ e-mail:monti@unizar.es
}

Received 28 June 2020; accepted 26 July 2020

\begin{abstract}
The classical and modified equations of Kolmogorov-Johnson-Mehl-Avrami are compared with the equations of conventional Gompertz and Montijano-Bergues-Bory-Gompertz, in the frame of growth kinetics of tumors. For this, different analytical and numerical criteria are used to demonstrate the similarity between them, in particular the distance of Hausdorff. The results show that these equations are similar from the mathematical point of view, and the parameters of the Gompertz equation are explicitly related to those of the Avrami equation. It is concluded that Modified Kolmogorov-Johnson-Mehl-Avrami and Montijano-Bergues-Bory-Gompertz equations can be used to describe the growth kinetics of unperturbed tumors.
\end{abstract}

Keywords: Avrami formulations; Gompertz formulations; tumor growth kinetics; fractal dimension.

PACS: 87.15.-v; 87.19.xj; 87.17.Rt; 07.05.Tp; 87.10.Ed; 05.45.Pq; 81.05.-t.

DOI: https://doi.org/10.31349/RevMexFis.66.632

\section{Introduction}

The classical Kolmogorov-Johnson-Mehl-Avrami (KJMA) and modified Kolmogorov-Johnson-Mehl-Avrami (mKJMA) equations are used firstly to fit experimental data of untreated Sa-37 fibrosarcoma tumor that grows in BALB/c/Cenp mice and agree with the Gompertz and Logistics conventional equations [1]. Recently, it has been proposed a new equation to describe data of the unperturbed Ehrlich and Sa-37 fibrosarcoma tumors based on the conventional Gompertz (CG) equation, named Montijano-Bergues-Bory-Gompertz (MBBG) equation (we refer the reader to see equations (8) and (9) in [2]).

The parameters of the CG equation are related to biological processes and the tumor fractal properties. The parame- ters of Avrami formulations (KJMA and mKJMA) have been associated with structural changes; diffusion, heterogeneity, and anisotropy processes; fractal, mechanical and electrical properties. The MBBG equation links explicitly the parameters of the CG equation with fractal dimensions of the tumor mass and its contour. The correspondence among these models guarantees that they may be indistinctly used to fit untreated tumor growth kinetics (TGK), and reveals cancer intrinsic findings. Additionally, this correspondence allows to explicitly relate parameters of each model. The later may reveal if the parameters of the CG equation are merely related to biological processes or other physical processes. Therefore, this study aims is to demonstrate the correspondence between these four Avrami and Gompertz formulations for TGK. Relationships between the coefficients of the KJMA 
and mKJMA equations and those of the CG and MBBG equations are reported.

\section{Models}

To demonstrate the correspondence between Avrami and Gompertz formulations, KJMA Eq. (1), mKJMA Eq. (2), CG Eq. (3) and MBBG Eq. (4) are used in this study.

$$
\begin{aligned}
& p(t)=1-e^{-(K t)^{n}}, \\
& p(t)=1-\left[1+(\lambda-1)(K t)^{n}\right]^{-1 /(\lambda-1)}, \\
& V(t)=V_{0} e^{\frac{\alpha}{\beta}\left(1-e^{-\beta t}\right)}, \\
& V(t)=V_{\text {obs }}\left(\frac{V_{0}}{V_{\text {obs }}}\right)^{e^{-\beta t}} e^{\frac{\alpha}{\beta}\left(1-e^{-\beta t}\right)} .
\end{aligned}
$$

Here $p(t)$ represents the fraction of cancer cells that pass to the solid tumor phase at time $t$ and $K, n$ and $\lambda$ parameters are tumor growth rate, Avrami coefficient, and impingement mechanisms, respectively. Parameters $V_{0}, V_{\text {obs }}$, and $V(t)$ represent the initial tumor volume at $t=0$, the tumor volume observed for its latency time, and the tumor volume at each instant of time $t$, respectively. The parameter $\alpha$ is interpreted as tumor growth rate, and $\beta$ has been connected with endogenous anti-angiogenesis mechanisms [1].

\subsection{Correspondence between Avrami and Gompertz formulations}

To establish the correspondence between Avrami and Gompertz formulations we must make a linear transformation to $V(t)$, becoming $q(t)$, so that it takes values between 0 and 1 , as $p(t)$ does. This may be argued because KJMA and mKJMA are normalized between 0 and 1, as reported in [1]. Then, $q(t)$ may be defined as

$$
q(t)=\frac{V(t)-V_{0}}{V(\infty)-V_{0}}
$$

where $V(t)$ may be any of two Gompertz formulations (CG and $\mathrm{MBBG}$ ), and $V(\infty)$ means mathematically the tumor volume as the time $t$ goes to infinity. By bio-ethical aspects, $V(\infty)$ coincides with the tumor volume that does not exceed $10 \%$ of the bodyweight [1-3].

The linear transformation performed guarantees that $p(t)$ and $q(t)$ have the same domain $[0, \infty)$ and the same set image $[0,1]$. So, it is possible to find some relationship between the parameters of these models so that their respective growth curves do not differ significantly. To measure the distance between the two curves, we will use the Hausdorff distance. Let $d(x, y)$ be a distance in $\mathbb{R}^{2}$ and consider the graph of functions $p(t)$ and $q(t)$ given by $\{(t, p(t)), t>0\}$ and $\{(\tau, q(\tau)), \tau>0\}$. The Hausdorff distance is defined as

$$
d_{H}(p, q)=\max \left\{d_{1}, d_{2}\right\}
$$

where

$$
\begin{aligned}
& d_{1}=\sup _{t>0} \inf _{\tau>0} d((t, p(t)),(\tau, q(\tau))), \\
& d_{2}=\sup _{\tau>0} \inf _{t>0} d((t, p(t)),(\tau, q(\tau))) .
\end{aligned}
$$

Since $p(t)$ and $q(t)$ have a point of inflection, to get close functions, we can study the conditions on the parameters of the functions for which they have the same inflection point, that is, at the same time $\tau p(\tau)=q(\tau)$ and for both functions, the second derivative vanishes at $t=\tau$. For CG equation we have

$$
\begin{aligned}
\frac{\mathrm{d}^{2}}{\mathrm{~d} t^{2}} q(t) & =0 \Rightarrow t=\tau=\frac{1}{\beta} \log \left(\frac{\alpha}{\beta}\right), \\
q^{\prime}(\tau) & =\frac{\beta e^{\alpha / \beta-1}}{e^{\alpha / \beta}-1}, \quad q(\tau)=\frac{-1+e^{\alpha / \beta-1}}{e^{\alpha / \beta}-1},
\end{aligned}
$$

while for MBBG equation

$$
\begin{aligned}
& \frac{d^{2}}{d t^{2}} q(t)=0 \Rightarrow t=\tau=\frac{1}{\beta} \log \left(\frac{\alpha}{\beta}-\log \left(\frac{V_{0}}{V_{\mathrm{obs}}}\right)\right), \quad q(\tau)=\frac{-V_{0}+e^{\alpha / \beta+\frac{\alpha}{-\alpha+\beta \log \left(V_{0} / V_{\mathrm{obs}}\right)} V_{\mathrm{obs}}\left(\frac{V_{0}}{V_{\mathrm{obs}}}\right)^{\frac{\beta}{\alpha-\beta \log \left(V_{0} / V_{\mathrm{obs}}\right)}}}}{V_{\mathrm{obs}} e^{\alpha / \beta}-V_{0}} \\
& q^{\prime}(\tau)=\frac{\beta e^{\alpha / \beta+\frac{\alpha}{-\alpha+\beta \log \left(V_{0} / V_{\mathrm{obs}}\right)}} V_{\mathrm{obs}}\left(\frac{V_{0}}{V_{\mathrm{obs}}}\right)^{\frac{\beta}{\alpha-\beta \log \left(V_{0} / V_{\mathrm{obs}}\right)}}}{V_{\mathrm{obs}} e^{\alpha / \beta}-V_{0}}
\end{aligned}
$$

Given fixed values of $\alpha, \beta$ (and $v_{0}, v_{\text {obs }}$ ) we obtain $\tau, q(\tau)$ and $q^{\prime}(\tau)$. It is required for KJMA to find values of $K$ and $n$ that make $p(\tau)=q(\tau)$ and $p(t)$ also has a point of inflexion at $\tau$. This implies that

$$
\frac{\mathrm{d}^{2}}{\mathrm{~d} t^{2}} p(t)=0 \Rightarrow t=\tau=\frac{1}{K}\left(\frac{n-1}{n}\right)^{1 / n},
$$

$$
p(\tau)=1-e^{-1+1 / n}=q(\tau),
$$

Eq. (8) and Eq. (7) give

$$
n=\frac{1}{1+\log (1-q(\tau))}, \quad K=\frac{1}{\tau}\left(\frac{n-1}{n}\right)^{(n-1) / n} \text {. }
$$


As $0<q(\tau)<1$, it follows that $n>0$ only if $1-q(\tau)<$ $1 / e$, and in this case, $n>1$.

As mKJMA equation has three parameters, it is assumed that $p(t)$ and $q(t)$ have a point of inflection at time $\tau$ such that $p(\tau)=q(\tau)$ and $p^{\prime}(\tau)=q^{\prime}(\tau)$ at same time $\tau$. Note that $\tau$, $q(\tau)$, and $q^{\prime}(\tau)$ can be expressed in terms of $\alpha, \beta, v_{0}$, and $v_{\text {obs }}$ parameters. Thus,

$$
\begin{aligned}
& \frac{\mathrm{d}^{2}}{\mathrm{~d} t^{2}} p(t)=0 \Rightarrow t=\tau=\frac{1}{K}\left(\frac{n-1}{n+\lambda-1}\right)^{1 / n} \\
& p(\tau)=1-\left(\frac{n \lambda}{n+\lambda-1}\right)^{-1 /(\lambda-1)}=q(\tau) \\
& p^{\prime}(\tau)=\frac{n-1}{\lambda} \frac{1}{\tau}(1-p(\tau))=q^{\prime}(\tau) .
\end{aligned}
$$

From Eq. (11) and Eq. (10) we deduce respectively that

$$
n=1+\lambda \frac{\tau q^{\prime}(\tau)}{1-q(\tau)}, \quad \frac{n \lambda}{n+\lambda-1}=\left(1-q(\tau)^{1-\lambda} .\right.
$$

Substituting the value of $n$ in the second equation yields

$$
1+\lambda \frac{\tau q^{\prime}(\tau)}{1-q(\tau)}-\frac{\left(1-q(\tau)+\tau q^{\prime}(\tau)\right)}{(1-q(\tau))^{\lambda}}=0
$$

which constitutes a non-linear equation in $\lambda$ of the form

$$
f(\lambda)=a+b \lambda+c^{\lambda}=0
$$

with $a=1 /\left(1-q(\tau)+\tau q^{\prime}(\tau)\right), b=\tau q^{\prime}(\tau) /(1-q(\tau))(1-$ $\left.\left.q(\tau)+\tau q^{\prime}(\tau)\right)\right)$ and $c=1 /(1-q(\tau))$.

Since $0 \leq q(t)<1$ and $q^{\prime}(t)>0$, then $c>1$ and $b>0$. This implies that $f(\lambda)$ tends to $-\infty$ when $\lambda$ tends to $\pm \infty$. Besides $f^{\prime}(\lambda)$ has a single root (corresponding to a maximum of $f$ and $f(1)=0 . f(\lambda)$ has exactly two roots on $\lambda$. If $b-c \log (c)>0$, another root of $f$ is greater than 1 . Otherwise, the other root would be less than 1 .

Given $\alpha, \beta, v_{\text {obs }}$ and $v_{0}$ (or $\alpha, \beta$ and $v_{0}$ ) such that

$$
\frac{\tau q^{\prime}(\tau)}{\left.(1-q(\tau))\left(1-q(\tau)+\tau q^{\prime}(\tau)\right)\right)}>-\frac{\log (1-q(\tau))}{1-q(\tau)},
$$

then there exist values of $n, \lambda$ and $K$ given by $\lambda$ solution of Eq. (12) greater than 1,

$$
n=1+\lambda \frac{\tau q^{\prime}(\tau)}{1-q(\tau)}, \quad K=\frac{1}{\tau}\left(\frac{n-1}{n+\lambda-1}\right)^{1 / n} .
$$

The above remarks reveal some fine aspects of this analysis. First, the condition that $p(t)$ and $q(t)$ have an inflection point at the same value $\tau$ is very restrictive. Second, given $q(t)$, it is not always possible to find a function $p(t)$ that meets the above conditions. Third, the function $p(t)$ obtained under the above requirements does not have to be closer enough to the given function $q(t)$. These difficulties disappear entirely if we search for the parameters that make minimum the Hausdorff distance $\min _{n, K, \lambda} d_{H}(p, q)$. In this case, the values of these parameters cannot be calculated analytically but be so numerically.

\subsection{Simulations}

For simulations, $\alpha=1.2$ days $^{-1}, \beta=0.2$ days $^{-1}, v_{0}=$ $0.05 \mathrm{~cm}^{3}$ and $v_{\text {obs }}=0.015 \mathrm{~cm}^{3}$ are used [2]. A computer program is implemented in the Matlab ${ }^{R}$ software (version R2012b 64-bit, Institute for Research in Mathematics and Applications, University of Zaragoza) to calculate numerically the values of $n, K$ and $\lambda$.

\section{Results}

Figures 1a) and 1b) show the comparison between MBBG (in red color) and KJMA (in black color) functions. Figure 1a) reveals that there is a small difference between these two functions for the value of $d_{H}(p, q)$ obtained from the values of $n$ and $K$ with the use of Eq. (9), as shown in Table I. Nevertheless, both functions coincide when $d_{H}(p, q)$ is minimal, see Fig 1b), a condition that is reached for the values of $n$ and $K$ displayed in Table I. Besides Figs. 1c) and 1d) reveal that there is a good agreement between MBBG (in red color) and mKJMA (in black color) functions. Figure 1c) is obtained for values of $n, K$ and $\lambda$ obtained from Eq. (13) (Table I). These two functions are practically identical when $d_{H}(p, q)$ is minimized (Fig. 1d)), resulting $n, K$ and $\lambda$ values that are exhibited in Table I.

Results of Fig. 1 and Table I are valid for the values of $\alpha, \beta$ and $V_{\text {obs }}$ fix in MBBG equation. Nevertheless, they can be numerically validated for any permissible value of each of these four parameters. Therefore, range of values of $\alpha \in[0.1,4], \beta \in[0.1,1]$ and $V_{\text {obs }} \in[0.01,0.1]$ are also used in this study. The most unfavorable case occurs for the maximum value of the minimum distance of $d_{H}(p, q)[4,5]$, given by

TABLE I. Hausdorff distances between the curves. Hausdorff distances between the MGE curve given by the parameters $\alpha=4.0$ days ${ }^{-1}$, $\beta=0.1$ days $^{-1}, v_{0}=0.05 \mathrm{~cm}^{3}, v_{\mathrm{obs}}=0.15 \mathrm{~cm}^{3}$ and the corresponding KJMA and mKJMA curves.

\begin{tabular}{ccccc}
\hline Curve & $d_{H}\left(\mathrm{~cm}^{3}\right)$ & $n$ & $K\left(\right.$ days $\left.^{-1}\right)$ & -1 \\
\hline KJMA from $(9)$ & 0.07179 & 1.84450 & 0.07136 & - \\
KJMA with minimum $d_{H}$ distance & 0.02585 & 2.20672 & 0.07139 & 0.08199 \\
mKJMA from (13) & 0.01423 & 2.87823 & 0.07954 & 1.64668 \\
mKJMA with minimum $d_{H}$ distance & 0.00265 & 2.74564 & 1.45667 \\
\hline
\end{tabular}




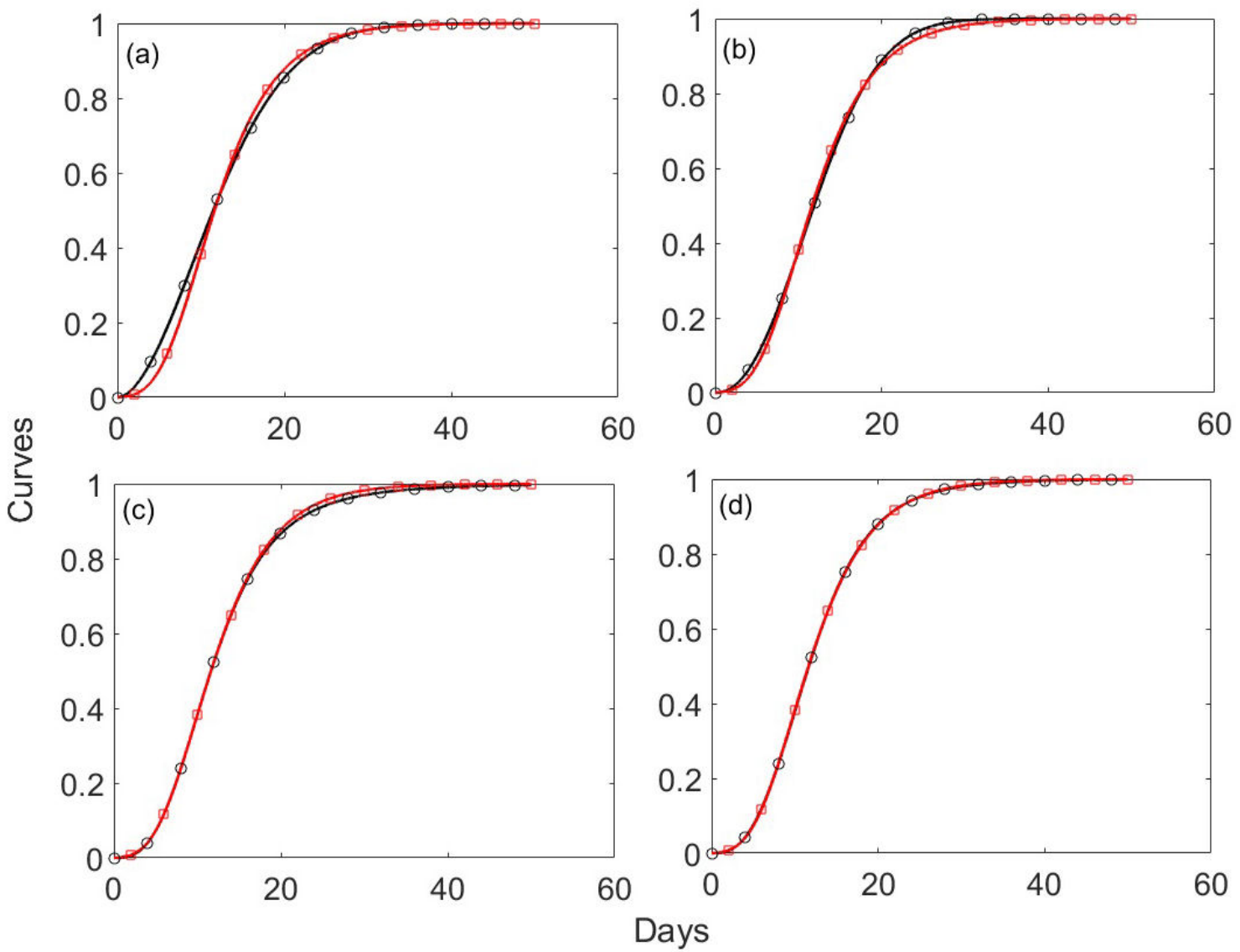

FIGURE 1. a) KJMA and MBBG equations according to Eq. (9). b) KJMA and MBBG equations with minimal Hausdorff distance. c) mKJMA and MBBG equations according to Eq. (13). d) mKJMA and MBBG equations with minimal Hausdorff distance. MBBG equation is identified in red color whereas both Avrami equations are displayed in black color. Simulations are made for $\alpha=1.2 \mathrm{days}{ }^{-1}$, $\beta=0.2$ days $^{-1}, v_{0}=0.05 \mathrm{~cm}^{3}$ and $v_{\mathrm{obs}}=0.15 \mathrm{~cm}^{3}$

$$
\max _{\alpha, \beta, V_{0}, V_{\text {obs }}} \min _{\lambda, K, n} d_{H}(p, q)
$$

This maximum value is $d_{H}(p, q)=0.00975 \mathrm{~cm}^{3}$, obtained for $\alpha=4.0$ days $^{-1}, \beta=0.1$ days $^{-1}, V_{0}=$ $0.05 \mathrm{~cm}^{3}, \quad V_{\text {obs }}=0.15 \mathrm{~cm}^{3}, \lambda=2.468, K=$ 0.0875 days $^{-1}$ and $n=2.689$.

\section{Discussion}

Numerical results confirm the fractal nature of the Gompertz and Avrami formulations, as in [2,7]. Besides these results demonstrate the existence of a good correspondence between them, and their parameters are closely related, unprecedented in the literature. Despite this, the KJMA equation cannot be used in the experiment because $d_{H}>0.05 \mathrm{~cm}^{3}$ (minimum mensurable volume), the value from which there are significant differences for the tumor volume, in agreement with the experiment [1].

Simulations demonstrate that $d_{H}(p, q)$ increases when the ratio $\alpha / \beta$ increases so that the most unfavorable case is obtained when $\alpha$ is maximum and $\beta$ is minimum, meaning that TGK is an exponential and not a sigmoid, in contrast with the experiment $[1-3,6]$ studies. From biological point of view, this means that the solid tumor grows instantaneously, and its size would be very large respect to organism size, an aspect not observed in preclinical and clinical studies. For this extreme case, the variation of $d_{H}(p, q)$ is not affected when $V_{\text {obs }}$ is varied in the range above-mentioned.

The results of this study reveal for the first time in the literature that: 1) there is a close relationship among $n, K, \lambda, \alpha, \beta, V_{\mathrm{obs}} / n_{\mathrm{obs}}$, cell loss mechanisms, the mass and the contour fractal dimensions of the tumor, indicating that $n, K$ and $\lambda$ depend on the tumor fractal dimension. 2) $\alpha$ and $\beta$ kinetic parameters are not biological in nature but they and TGK are governed by tumor mechanical properties (i.e., Young modulus), heterogeneity, anisotropy, heterogeneous nucleation mechanisms, change of geometry and dynamic structural transformations that happen during the tumor growth. This may suggest that $\alpha$ and $\beta$ kinetic parameters, angiogenesis process, heterogeneity, anisotropy, hardness, irregular shape and edge, cell multiplication, metastasis, tumor evasion mechanisms to the immune system, cell loss mechanisms and other biological parameters involved during formation and growth of a tumor emerge depending on how the electrical and mechanical properties of the tumor are 
changing in time. All these aspects may be interrelated taking into account the results of $[1,2]$. The parameters $n, K$ and lambda in mKJMA are related with tumor structural changes when this equation is used to describe Ehrlich and fibrosarcoma Sa-37 TGK [1]. Besides it is explicitly demonstrated that the parameters alpha and beta in the MBBG equation depend on $V_{\text {obs }} / n_{\text {obs }}$, cell loss mechanisms, the mass, and the contour fractal dimensions of the tumor (we refer the reader to see Eqs. (9) for alpha and beta in [2]). As a result of these associations among the parameters of MBBG with KJMA and mKJMA, these non-linear and complex processes may partially explain why current targeted cancer therapies have not given the expected objective response (cancer complete cure or its conversion into a chronic disease), in agreement with $[1,2,7-11]$.

Many experimental studies have been demonstrated that the Gompertz model is more feasible to fit the tumor growth kinetics. This finding has been proved in different tumor histological varieties and host types [12-15]. That is why, we do not use the Logistic equation in this study. Nevertheless, Avrami coefficients may be related to those of other Gompertz formulations following the ideas of this study. For this, we must previously demonstrate that these formulations adequately fit the experimental data that follow a sigmoidal behavior. In principle, the results of this study may be extended to any biological systems (for example, microorganisms) or non-biological system (for example, crystals), whose growth kinetics are sigmoid.

It is expected that $n, K, \lambda, \alpha$ and $\beta$ parameters and the mass and contour fractal dimensions of the tumor involve during the entire TGK are not constant in time but depend on it. A further study is required to establish the temporal dependence of each of these parameters. This may be transcendent for chemotherapy and immunotherapy. Additionally, this study and $[1,2]$ add new contributions towards a better understanding of TGK and provides the basis to propose a personalized physical therapy addressed to the mechanical/electrical properties of cancer. In conclusion, there exists a good correspondence among mKJMA and MBBG equations from a theoretical point and each parameter $n, K$ and $\lambda$ of mKJMA equation depend on $\alpha$ and $\beta$ parameters, $V_{\mathrm{obs}} / n_{\mathrm{obs}}$, cell loss mechanisms as well as the mass and the contour fractal dimensions of the tumor involved in the MBBG equation.

\section{Acknowledgments}

Authors thank the valuable help and useful comments of unknown reviewers and Editor in Chief. This work has been partially supported by the Oriente University, Cuba, under the grants \# 9494 (FITIB) and \# 9116 (CNEA), and MINECO, Spain, under the Project MTM2016-77735-C3-1-P.
1. M. M. González et al., Is cancer a pure growth curve or does it follow a kinetics of dynamical structural transformation?, BMC Cancer 17 (2017) 174, https://doi.org/ $10.1186 / \mathrm{s} 12885-017-3159-y$

2. A. R. S. Castañeda et al., New formulation of the Gompertz equation to describe the kinetics of untreated tumors, Plos One 14 (2019) e0224978. https://doi.org/10.1371/ journal.pone.0224978

3. M. M. González et al., Dose-response study for the highly aggressive and metastatic primary F3II mammary carcinoma under direct current. Bioelectromagnetics 39 (2018) 460. https : //doi.org/10.1002/bem.22132

4. CS273: Algorithms for Structure and Motion in Biology, (Stanford University, Stanford, California, 2004). Available from: http://web.stanford.edu/class/cs273/ scribing/2004/class8/scribe8.pdf

5. D. P. Huttenlocher, G. A. Klanderman, and W. J. Rucklidg Comparing images using the Hausdorff distance. IEEE Trans. Pattern Anal. Mach. Intell. 15 (1993) 850.

6. N. Olea, M. Villalobos, M. I. Nunez, J. Elvira, J. R. de Almodovar, and V. Pedraza, Evaluation of the growth rate of MCF7 breast cancer multicellular spheroids using three mathematical models. Cell proliferation 27 (1994) 213. https : / / doi. org/10.1111/j.1365-2184.1994.tb01418.x

7. M. Molski, and J. Konarski Coherent states of Gompertzian growth. Phys. Rev. E 68 (2003) 021916. https://doi. org/10.1103/PhysRevE.68.021916
8. C. Voutouri, F. Mpekris, P. Papageorgis, A. D. Odysseos, and T. Stylianopoulos, Role of constitutive behavior and tumor-host mechanical interactions in the state of stress and growth of solid tumors. PloS One 9 (2014) e104717. https: / / doi.org/ $10.1371 /$ journal.pone.0104717

9. A. Rubiano et al., Viscoelastic properties of human pancreatic tumors and in vitro constructs to mimic mechanical properties. Acta biomaterialia 67 (2018) 331. https: / / doi .org/10 . $1016 / j . a c t b i o .2017 .11 .037$

10. J. Fenner, A. C. Stacer, F. Winterroth, T. D. Johnson, K. E. Luker, and G. D. Luker Macroscopic stiffness of breast tumors predicts metastasis. Sci. Rep. 4 (2014) 5512. https: //doi.org/10.1038/srep05512

11. C. Voutouri and T. Stylianopoulos Accumulation of mechanical forces in tumors is related to hyaluronan content and tissue stiffness. PloS One 13 (2018) e0193801. https : / / doi. org/10.1371/journal.pone.0193801

12. V. G. Vaidya and F. J. Alexandro, J. Biomed. Comput. 13 (1982) 19. DOI: $10.1016 / 0020-7101$ (82) 90048-4

13. M. Marušic, Z̆. Bajzer, and S. V. Vuk-Pavlovic, Bull. Math. Biol. 56 (1994) 617. https://doi.org/10.1007/ BF 02460714

14. M. Marušic, Math. Commun. 1 (1996) 175.

15. D. Miklavčič, T. Jarm, R. Karba, and G. Serša, Math. Simul. Comp. 39 (1995) 597. 